joint replacement surgery. ASCs were isolated and cultured with/ without human recombinant leptin, TNF, adiponectin (LMW, HMW) and IFN $\gamma$. After $24 \mathrm{~h}$, secretion of cytokines was measured by ELISA. Conditioned media from ASCs cultures were used to stimulate FLS. After stimulation, FLS proliferation and apoptosis was determined (by incorporation of $\mathrm{BrdU}$ and flow cytometry, respectively). Cytokine concentration in FLS culture supernatants was determined by ELISA. The Wilcoxon signed-rank test was used for statistical analysis.

Results HMW adiponectin enhanced IL-6, IL-8, VEGF $(p<0.001)$ and TGF $\beta(p<0.01)$ production. LMW adiponectin increased secretion of IL- 6 and VEGF $(p<0.001)$, but its influence was much weaker than HMW's. TNF was the most potent in stimulating IL-6 and IL-8 production by ASCs $(\mathrm{p}<0.001)$. The influence of adipocytokine treated-ASC-conditioned media on FLS was assesed by comparison with two controls: untreated ASC-conditioned medium and control FLS medium. ASC-conditioned medium treated with HMW caused down-regulation of IL- 6 secretion by FLS comparing to both controls $(p<0.05)$. TNF-treated ASC-conditioned medium induced significant increase in MMP-3 production by FLS comparing to both controls $(p<0.05)$. Proliferation of FLS was up-regulated after untreated ASC-conditioned medium $(p<0.01)$ and this was partly reversed by HMW and TNF-treated ASC-conditioned media $(p<0.05)$. HMW-treated ASC-conditioned medium increased percentage of FLS in late apoptosis $(\mathrm{p}<0.05)$.

Conclusions HMW and TNF seem to be the most potent in altering RA-ASCs properties. Leptin had no effect and LMW exerted only slight effect. HMW was able to up-regulate factors thought to mediate ASCs immunosupression (TGF $\beta$, IL-6). In addition, its effect on FLS shows that this adipokine may exert positive effect on immunosupressive ASCs function. However, because HMW upregulated also proangiogenic cytokines (IL-8, VEGF), it is difficult to determine its real impact on RA-ASCs. TNF role in modulating RAASCs also need to be elucidated.

Funding Grant No. N/NZ5/00932, National Science Center, Poland.

\section{B cells and autoimmunity}

\section{A5.1 ABNORMAL CALCIUM INFLUX IN T AND B LYMPHOCYTES FROM SYSTEMIC LUPUS ERYTHEMATOSUS PATIENTS IS RELATED TO STIM-1 OVER-EXPRESSION}

doi:10.1136/annrheumdis-2013-203219.1

${ }^{1} Y$ ves Renaudineau, ${ }^{2}$ Olivier Mignen, 'Tinhinane Fali, ${ }^{2}$ Miguel Burgos, 'Divi Cornec 'Sandrine Jousse, 'Alain Saraux, 'Jacques-Olivier Pers. 'EA2216 Immunology, Pathology and Immunotherapy, Brest University and Brest Medical School Hospital, Brest, France; 2INSERM U613, Faculty of Medicine, Brest, France

Background and Objectives Recently described, the molecule STIM1 (stromal interaction molecule 1) acts as a key mediator of calcium influx by controlling cell proliferation after antigen stimulation, Erk phosphorylation, cytokine production and apoptosis. Although STIM1 mutations have been associated with severe immunodeficiency, no study has focused on the STIM1 molecule in autoimmune diseases.

Materials and Methods $\mathrm{T}$ and $\mathrm{B}$ lymphocytes, purified by negative selection from peripheral blood of patients with systemic lupus erythematosus (SLE, $\mathrm{n}=11$ ), rheumatoid arthritis (RA, $n=7)$, primary Sjögren's syndrome ( $\mathrm{pSS}, \mathrm{n}=11)$ and healthy controls $(\mathrm{HC}, \mathrm{n}=12$ ) were tested by flow cytometry and Western blotting to determine the expression of STIM1. Video microscopy using specific probes has been used to assess intracellular calcium levels.
Results T cells from peripheral blood of HC express more STIM1 molecules (mean fluorescence intensity (MFI) $3.42 \pm 0.13$ ) than B cells (MFI $2.18 \pm 0.20, \mathrm{P}<0.01$ ). In B lymphocyte subpopulations, the expression of STIM1 is 2 times higher in $\mathrm{CD} 24^{\text {high }} \mathrm{CD} 38^{\text {high }}$ transitional B cells (MFI $4.83 \pm 0.63$ ) compared with CD24 ${ }^{\text {low }} \mathrm{CD} 38^{\text {low }}$ mature B cells (MFI $2.47 \pm 0.15, \mathrm{P}<0.01)$ and $\mathrm{CD} 24^{\text {high }} \mathrm{CD} 38^{\text {low }}$ memory B cells (MFI $3.64 \pm 0.42, \mathrm{P}<0.05$ ). The expression of STIM1 in $\mathrm{T}$ and $\mathrm{B}$ lymphocytes from patients with RA and $\mathrm{pSS}$ was similar to HC.

An highest calcium influx and a constitutive Erk phosphorylation characterise $\mathrm{T}$ and $\mathrm{B}$ cells from SLE patients when compared with HC and disease controls. As suspected, STIM1 is overexpressed in SLE, when compared with $\mathrm{HC}$ and this expression is similar between T cells (MFI $8.70 \pm 0.87$ ) and B cells (MFI $9.00 \pm$ 1.08). Within B cell subsets, STIM1 expression is 3.4 fold highest in transitional SLE B cells (MFI: $16.25 \pm 2.18, P<0.001$ ) than in transitional HC B cells and 2.3 fold highest than in mature SLE B cells (MFI: $7.1 \pm 1.53$ ) and memory SLE B cells (MFI: $9.51 \pm 2.01$ ). Western blotting results confirm the highest expression of STIM1 in SLE. Transient transfection of STIM1-targeting siRNAs was shown to restore the calcium influx and decrease Erk phosphorylation. Of particular note, the associations of $\mathrm{CpG} / \mathrm{anti-IgM} \mathrm{Ab}$ and $\mathrm{CpG}$ /anti-CD40 $\mathrm{Ab}$ are effective to induce STIM1 expression. Finally, STIM1 level was not correlated with the SLE disease activity index (SLEDAI) and autoantibodies (ANA, anti-dsDNA, anti-SSA/Ro).

Conclusions These results suggest that the differential expression of STIM1 may be an important factor in the process of lymphocyte self-reactivity in SLE, which opens new pathophysiological and therapeutic perspectives.

\section{A5.2 ACCUMULATION OF CIRCULATING AUTOREACTIVE NAIIVE B CELLS REVEAL DEFECTS OF EARLY B CELL TOLERANCE CHECKPOINTS IN PATIENTS WITH SJÖGREN'S SYNDROME}

doi:10.1136/annrheumdis-2013-203219.2

'Elisa Corsiero, 'Nurhan Sutcliffe, ${ }^{2}$ Hedda Wardemann, 'Costantino Pitzalis, 'Michele Bombardieri. 'Centre for Experimental Medicine \& Rheumatology, William Harvey Research Institute, Barts and The London School of Medicine \& Dentistry, Queen Mary University of London, John Vane Science Centre, Charterhouse Square, London EC1M 6BO, UK; ${ }^{2}$ Max Planck Institute for Infection Biology, 10117 Berlin, Germany

Background and Objectives Sjögren's syndrome (SS) is an autoimmune disease characterised by high affinity circulating autoantibodies and peripheral $\mathrm{B}$ cell disturbances with predominance of naive and reduction of memory $B$ cells. The stage at which errors in $\mathrm{B}$ cell tolerance checkpoints accumulate in SS is unknown. Here we determined the frequency of self- and poly-reactive B cells in the circulating naïve compartment of SS patients.

Materials and Methods Single CD27-IgD + B cells were sorted by FACS from peripheral blood of SS patients and healthy donors (HD). RNA was used to amplify Ig VH and VL genes and PCR products were cloned and expressed as recombinant monoclonal antibodies displaying identical specificity of the original B cells. Recombinant antibodies were tested towards different antigens to determine the frequency of autoreactive and polyreactive clones.

Results 66 recombinant antibodies were generated from naïve B cells of 4 SS patients and compared to 45 clones from $2 \mathrm{HD}$. Analysis of the VH and VL gene usage showed no significant differences between SS and HD. Conversely, we observed accumulation of circulating autoreactive naïve B cells in SS as demonstrated by increased reactivity towards Hep2 cells (43.1\% SS versus $25 \% \mathrm{HD}$ ) and ENA (19.6\% SS clones versus none). Among ENA+ clones, 6 displayed reactivity towards Ro/SSA and/or La/SSB. 
Conclusions Here using an efficient strategy to express recombinant monoclonal antibodies from single B cells we demonstrated an elevated frequency of autoreactive naïve $B$ cells in the circulation of SS patients supporting the existence of early defects in B cell tolerance checkpoints in SS.

\section{A5.3 ALTERATIONS ON PERIPHERAL BLOOD B CELL COMPARTMENTS IN SYSTEMIC LUPUS ERYTHEMATOSUS: RELEVANCE FOR MONITORING LUPUS ACTIVITY AND THERAPY}

doi:10.1136/annrheumdis-2013-203219.3

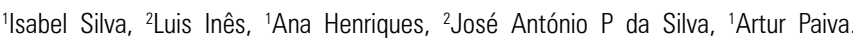
${ }^{1}$ Cytometry Service of the Blood and Transplantation Center of Coimbra, Portugal; ${ }^{2}$ Rheumatology Department; Centro Hospitalar e Universitário e Coimbra, Portugal

Background and Objectives Despite recent insights on abnormalities of blood B cell subsets in human systemic lupus erythematosus (SLE), a peripheral blood biomarker with useful clinical information about the occurrence of an active disease period hasn't yet been achieved. Moreover, the clinical relevance of anti-dsDNA antibodies and their utility for monitoring an individual patient remains a matter of debate. In this sense, we attempt to determine whether the degree of abnormalities of circulating B cell subsets correlates with SLE disease activity and constitute an useful tool for SLE patients monitoring.

Materials and Methods We analysed by flow cytometry the major circulating B cell subsets (immature, naïve, memory and plasmablast) and their expression profile of $B$ cell related molecules (CD19, CD20, CD81 and BAFFR) in 43 SLE patients, 18 with active and 25 with inactive disease, according to the SLE Disease Activity Index 2000 (SLEDAI, 2k), as well as in 30 healthy individuals.

Results The results pointed to the existence of significant alterations on B cell homeostasis that are significantly correlated with disease activity. An overall decrease in absolute numbers of all B cell subsets was observed in SLE patients, with the exception of IgGplasmablast that remained equal or even higher than in the control group, particularly in active disease. Additionally, a higher number of plasmablast expressing each Ig-heavy chain isotypes was found in patients with mucocutaneous involvement. Moreover, among memory B cells, an increased IgG and decreased IgM positive cellswas observed in both SLE groups.

Furthermore, a decreased expression of CD19 observed in active disease and an increased BAFFR expression in inactive disease in the majority of $B$ cell subsets, may contribute not only for breaking tolerance during $\mathrm{B}$ cell development, but also for enhancing plasmablast survival.

Conclusions In conclusion, flow cytometric monitoring of circulating B cell subsets, particularly focused on relative and absolute numbers of IgG plasmablasts, could provide a useful tool for monitoring disease activity, but also the therapy efficacy in patients with SLE.

\section{A5.4 ANTI CARBAMYLATED PROTEIN ANTIBODIES (ANTI-CARP) ARE PRESENT IN ARTHRALGIA PATIENTS AND PREDICT THE DEVELOPMENT OF RHEUMATOID ARTHRITIS}

doi:10.1136/annrheumdis-2013-203219.4

1,* Jing Shi, ${ }^{2, *}$ Lotte A van de Stadt, 'EW Nivine Levarht, 'Tom WJ Huizinga, 'René EM Toes, 'Leendert A Trouw, ${ }^{3}$ Dirkjan van Schaardenburg. 'Leiden University Medical Center, Leiden; '2Jan van Breemen Research Institute| Reade, Sanquin Research, Amsterdam; 3Jan van Breemen Research Institute | Reade and VU University Medical Center, Amsterdam, The Netherlands

*Mr. Shi and Dr. van de Stadt contributed equally to this work.

Background/Objective Recently, we discovered a new autoantibody system in rheumatoid arthritis (RA): anti carbamylated protein antibodies (anti-CarP). These antibodies have additional prognostic value in predicting joint destruction when compared to anti-citrullinated protein antibodies (ACPA). However, it is not yet known whether anti-CarP antibodies are present before the diagnosis of RA and whether they have predictive value for the development of RA. Therefore we studied whether anti-CarP antibodies are present in arthralgia patients and whether their presence associates with the development of RA.

Methods Sera of 340 arthralgia patients without clinical signs of arthritis and 32 healthy controls were measured for the presence of anti-CarP IgG antibodies. One hundred eleven arthralgia patients $(33 \%)$ were IgM-rheumatoid factor (IgM-RF) positive/anti-cyclic citrullinated peptide 2 (aCCP2) negative and 229 (67\%) were aCCP2 positive. Patients were followed for the development of RA (2010 criteria). The median follow up time was 36 months. Cox regression analysis was performed to compare the risk of developing RA between Anti-CarP positive and negative arthralgia patients in follow up time.

Results The arthralgia cohort consisted of 340 IgM-RF and/or aCCP positive patients. Anti-CarP antibodies were present in sera of $113(39 \%)$ of the tested patients. A total of 120 patients developed RA after a median (IOR) of 12 (6-24) months. The presence of antiCarP antibodies was associated with the development of RA in the whole arthralgia cohort even after correction for RF and aCCP2 status (HR: 1.56; 95\%CI: 1.06-2.29; $\mathrm{p}=0.023$ ), as well as in the aCCP2 positive subgroup (OR: 2.231; 95\%CI: 1.31-3.79; $p=0.003$ ).

Conclusions Anti-CarP antibodies were present in arthralgia patients and their presence predicted the development of RA independent of aCCP2 antibodies.

Disclosure These studies were financially supported by Janssen Biologics BV, (Johnson \& Johnson).

\section{A5.5 ANTIBODIES AGAINST DOMAIN I OF $\boldsymbol{\beta} 2$ GLYCOPROTEIN I IN ANTIPHOSPHOLIPID ANTIBODY SYNDROME}

doi:10.1136/annrheumdis-2013-203219.5

${ }^{1-3}$ Cecilia Chighizola, ${ }^{1}$ Francesca Pregnolato, 'Claudia Grossi, ${ }^{2,3}$ Maria Gerosa, ${ }^{4}$ Gary Norman, ${ }^{4}$ Michael Mahler, ${ }^{1,2}$ Maria Orietta Borghi, ${ }^{1-3}$ Pier Luigi Meroni. 'IRCCS Istituto Auxologico Italiano, Milan, Italy; ${ }^{2}$ University of Milan, Italy; ${ }^{3}$ stituto G Pini, Milan, Italy; ${ }^{4}$ Inova Diagnostics, Inc., San Diego, CA, USA

Background and Objectives antibodies anti-phospholipids (aPL) react to proteins bound to $\mathrm{PL}$, mainly $\beta 2$ glycoprotein I ( $\beta 2 \mathrm{GPI}$ ). Antibodies against $\beta 2 \mathrm{GPI}$ (a $\beta 2 \mathrm{GPI}$ ) exert a pathogenic role and represent a risk-factor for clinical manifestations of anti-phospholipid syndrome (APS). However, some aß2GPI-positive subjects never develop APS-related clinical manifestations. This observation may be explained by the heterogeneity of a $\beta 2 \mathrm{GPI}$ population, with autoantibody subgroups targeting different $\beta 2 \mathrm{GPI}$ epitopes. In particular, antibodies anti-domain I (aDI) but not domains IV and V $(\mathrm{aDIV} / \mathrm{V})$ of $\beta 2 \mathrm{GPI}$ have been associated with thrombotic events. Therefore, the aim of this study was to assess the prevalence of aDI and aDIV/V IgG in a cohort of aPL-positive patients.

Material and Methods 58 patients with a diagnosis of primary APS (PAPS) according to the 2006 Sydney criteria have been included in this study. 38 PAPS patients (65.5\%) presented with venous and/ or arterious thrombothic events while 20 subjects $(34.5 \%)$ had obstetric manifestations only. $15 \mathrm{aPL}$ asymptomatic carriers were also recruited. All samples had been tested for LA and for aCL and a 32 GPI with home-made assays according to international guidelines. In the thrombotic PAPS group, 35/38 subjects (92.1\%) were aß2GPI IgG positive; a 32 GPI IgG positivity rate was $85 \%$ in the obstetric PAPS group (17/20 women); $80 \%$ of the asymptomatic aPL carriers displayed a $\beta 2$ GPI IgG. IgG specificities against whole $\beta 2 \mathrm{GPI}$, $\mathrm{DI}$ and DIV/V have been evaluated with a novel solid-phase chemiluminiscent assay (BioFlash and ELISA, INOVA Diagnostics). 